\title{
ESTIMATIVA DO CONSUMO DE ÁGUA NA CULTURA DA CENOURA (Daucus carota, L.) v. NANTES SUPERIOR, PARA A REGIÃo de PIRACICABA, ATRAVÉS DO MÉTODO DO BALANÇO HÍDRICO
}

\author{
M.V.T. MOURA \\ Secretaria de Abastecimento/Centro Administrativo/SA. BR 101, km 0 - CEP: 59000-000-Lagoa Nova, RN \\ S. MARQUES JÚNIOR \\ Faculdade de Agronomia Dr. Francisco Maeda - Rua Cel. Flauzino B. Sandoval, 1259-CEP: 14500-000 - Ituverava,SP \\ T.A. BOTREL; J.A. FRIZONE \\ Departamento de Engenharia Rural-ESALQ/USP, C.P. 9, CEP: 13418-900 - Piracicaba,SP
}

\begin{abstract}
RESUMO: $O$ presente trabalho teve como objetivo estimar a demanda hídrica da cultura da cenoura (Daucus carota, L.) cr. Nantes Superior, pelo método do balanço hídrico sob condiçóes de campo, para a região de Piracicaba, Estado de São Paulo (22 42' 30" S; 47 30' 00' W, à 560 metros de altitude). Os valores de evapotranspiração máxima obtidos pelo método do balanço hídrico foram correlacionados com os valores de evapotranspiração de referência estimados pelo método de Penman, objetivando a determinação do coeficiente de cultura. 0 consumo total de água considerando-se um ciclo vegetativo de 101 dias foi de $365 \mathrm{~mm}$, gerando um consumo médio de 3,61 $\mathrm{mm} /$ dia. Nesta situação, o coeficiente de cultura médio encontrado foi de 1,1.
\end{abstract}

Descritores: cenoura, consumo de água, balanço hídrico.

\section{ESTIMATION OF CARROT WATER CONSUMPTION (Daucus carota, L.) V. NANTES SUPERIOR, IN PIRACICABA COUNTY THROUGH THE WATER BALANCE METHODOLOGY}

\begin{abstract}
To estimate the evapotranspiration of a carrot crop (Daucus carota, L.) v. Nantes Superior, under field conditions, in Piracicaba $\left(22^{\circ} 42^{\prime} 30^{\prime}\right.$ ' $\mathrm{S} ; 47^{\circ} 30^{\prime} 00^{\prime}$ ' W, at $560 \mathrm{~m}$.), maximum evapotranspiration was determinated by the water balance method and correlated to the reference evapotranspiration estimated by Penman's method, in order to obtain the crop coefficient. The total water consumption of the vegetative cycle (101 days) was about 365 $\mathrm{mm}$, with a mean of $3,61 \mathrm{~mm} /$ dia. In this situation the mean crop coefficient was 1.1 .
\end{abstract}

Key Words: carrot, water consumption, water balance.

\section{INTRODUÇÃO}

O crescimento da população mundial não deixa dúvidas quanto a necessidade de um aumento substancial na produção de alimentos. Neste contexto, a premissa de que a produção vegetal é o resultado de relações interdependentes do sistema solo-atmosfera com a planta, leva a concluir que é indispensável o estudo de diversos fatores correlacionados nesse meio, quando se procura o aumento da produtividade agrícola.

Os elementos climáticos são variáveis com o tempo, fazendo com que a exploração agrícola esteja sempre sujeita à incertezas. Dentre os fatores meteorológicos, a precipitação apresenta grande variabilidade espaço-temporal, cuja estimativa constitue-se uma das principais fontes de erro em planejamentos agropecuários.
Face à importância para a agricultura do conhecimento do balanço de água de uma região, cujos valores são potencialmente determinados pela evapotranspiração, inúmeros trabalhos foram desenvolvidos objetivando quantificar parâmetros envolvidos nesta estimativa. Entre as diversas metodologias existentes, o balanço hídrico num volume de controle de solo e fundamental do ponto de vista agronômico, pois define as condições hídricas nas quais as culturas se desenvolvem (REICHARDT, 1985).

Esta forma de estimativa tem sido utilizado por diversos pesquisadores na determinação do consumo hídrico das culturas (ROSE \& STERN, 1969; REICHARDT et al., 1989; SAAD, 1991, entre outros). A metodologia empregada baseia-se na somatória das quantidades de água que entram e saem de um deteminado 
elemento de volume de solo, num dado intervalo de tempo. Como resultado tem-se a quantidade de água líquida disponivel as plantas.

Os componentes desse método são apresentados na equação geral do seguinte balanço de massa:

$$
\begin{aligned}
& \int_{t_{0}}^{t 1}(p+i-d s-e \pm q z) \cdot d t= \\
& =\int_{0}^{L} \int_{t o}^{t 1}\left(\frac{\delta \theta}{\delta t}\right) \cdot d t \cdot d z \quad(1)
\end{aligned}
$$

onde $p, i, d s, e, e$ qz representam os fluxos de precipitação, irrigação, deflúvio superficial, evapotranspiração, drenagem profunda $(-)$ ou ascenção capilar $(+)$ e $\delta \Theta / \delta t$ a variação de armazenamento de água em uma camada de solo de comprimento $\mathrm{L}$, em um intervalo de tempo (t1-t0), respectivamente.

Para o planejamento de sistemas de irrigação há a necessidade do conhecimento de parâmetros edafo-climáticos locais, assim como características inerentes à cultura, de modo a determinar os riscos de perda de produção por deficiência hídrica. Assim sendo, para que se garanta o êxito na utilização da técnica da irrigação, é imprescindível o conhecimento da água a ser aplicada, bem como da frequência de aplicação desta, como comenta FRIZZONE (1986).

Embora seja recomendado o emprego de valores de coeficiente de cultura obtidos em condições locais, o número de informações disponiveis nos diversos centros de pesquisa do país são incipientes para recomendações definitivas.

Tal situação pode ser verificada quando se estuda a cultura da cenoura (Daucus carota, L.), uma das principais hortículas empregadas na alimentação brasileira, haja visto o seu valor nutritivo e medicinal, além de grande aceitação por parte do mercado consumidor. No Brasil, é produzida em uma área de aproximadamente $11 \mathrm{mil}$ hectares da qual cerca de $60 \%$ utiliza a irrigação (MAROUELLI \& VIEIRA,1990).

$O$ presente trabalho tem como objetivo a determinação do consumo de água na cultura da cenoura, nas condições climáticas de Piracicaba$\mathrm{SP}$, visando criar subsídios para uma planejamento mais criterioso no cultivo irrigado da cultura na citada região.

\section{MATERIAL E METODOS}

O presente estudo foi desenvolvido em área experimental do Departamento de Engenharia Rural, no campus da Escola Superior de Agricultura "Luiz de Queiroz", situada no município de Piracicaba, SP, cujas coordenadas geográficas são: latitude $22^{\circ} 42^{\prime} 30^{\prime}$ ' S, longitude $47^{\circ} 30^{\prime} 00^{\prime \prime} \mathrm{W}$, e altitude de 560 metros. O local do experimento constou de uma área com 7 metros de largura por 10 metros de comprimento, cuja declividade estimada foi de $1,5 \%$.

Esta área foi subdividida em 5 canteiros, onde cada canteiro era formado por 1 metro de largura por 10 metros de comprimento, construídos perpendicularmente ao maior declive do local, espaçados de 0,5 metros. Nesta situação, os canteiros apresentaram uma profundidade de 0,25 metros.

Solo: $O$ solo do local foi classificado por RANZANI et al. (1966) como Terra Roxa Estruturada série "Luiz de Queiroz", sendo enquadrado na classificação americana como um Oxic Palendalfic, cujos materiais de origem são as eruptivas básicas. A TABELA 1 apresenta o resultado da análise granulométrica do solo.

TABELA 1 - Análises físicas do solo.

\begin{tabular}{cccc}
\hline Camada & & \multicolumn{2}{c}{ Granulometria (\%) } \\
\cline { 2 - 4 }$(\mathrm{cm})$ & Areia & Silte & Argila \\
\hline $0-20$ & 39,43 & 17,78 & 45,79 \\
$20-40$ & 40,55 & 15,69 & 43,76 \\
\hline
\end{tabular}

FONTE: Laboratório de Solos - DER-ESALQ/USP

Para a determinação da curva de retenção da água foram coletadas amostras indeformadas de solo através de anéis volumétricos de $54 \mathrm{~cm}^{3}$ para as profundidades de $0-10,10-20,20-30$ e $30-40 \mathrm{~cm}$, com cinco repetições. As amostras foram previamente saturadas em laboratório e submetidas à processo de secagem com tensões que variaram de 0,001 a 1,5 $\mathrm{MPa}$, sendo utilizados para esse processo a mesa de tensão e câmara de pressão de Richards.

Os valores de tensão obtidos para cada profundidade foram ajustados ao modelo proposto por VAN GENUTCHEN (1980), para obtenção da relação entre umidade e tensão da água no solo: 


$$
\theta=\theta r+\frac{(\theta s-\theta s)}{\left[1+(\alpha \cdot|h|)^{n}\right]^{m}} \quad(2:
$$

onde $\theta, \theta$ s e $\theta$ representam respectivamente, a umidade volumétrica atual, umidade volumétrica de saturação e umidade volumétrica residual, obtida à 1,5 $\mathrm{MPa}$. Os parâmetros $\alpha, \mathrm{m}$ e $\mathrm{n}$ cujos valores são dependentes do solo, foram obtidos a partir de regressão múltipla de pares de dados entre tensão e umidade, sendo apresentados na TABELA 2.

TABELA 2 - Parâmetros de ajuste da curva de retenção à equação de VAN GENUCHTEN (1980).

\begin{tabular}{|c|c|c|c|c|c|}
\hline Camadas & $\alpha$ & $\mathrm{m}$ & $\mathrm{n}$ & $\theta \mathbf{r}$ & $\theta \mathrm{s}$ \\
\hline$(\mathrm{cm})$ & & & & & $\left.\mathrm{cm}^{3}\right)$ \\
\hline
\end{tabular}

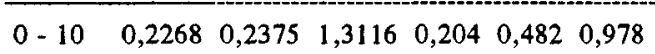

$\begin{array}{lllllll}10-20 & 0,2722 & 0,2107 & 1,2669 & 0,213 & 0,453 & 0,967\end{array}$

$\begin{array}{llllllll}20-30 & 0,1460 & 0,2012 & 1,2520 & 0,244 & 0,449 & 0,935\end{array}$

$\begin{array}{lllllll}30-40 & 0,1743 & 0,2049 & 1,2578 & 0,271 & 0,502 & 0,944\end{array}$

Para a determinação da condutividade hidráulica do solo saturado (Ks), foram coletadas amostras indeformadas utilizando-se o cilindro de Uhland (volume de $273 \mathrm{~cm}^{3}$ ), nas profundidades descritas anteriormente para a obtenção da curva de retenção, onde também foram utilizadas cinco repetições. Estas determinações foram feitas em laboratório através do método do permeâmetro de carga constante. Os resultados obtidos são apresentados na TABELA 3.

TABELA 3 - Condutividade hidráulica do solo saturado

\begin{tabular}{cccc}
\hline $\begin{array}{c}\text { Camada } \\
(\mathrm{cm})\end{array}$ & $\begin{array}{c}\mathrm{Ks} \\
(\mathrm{cm} / \mathrm{h})\end{array}$ & $\begin{array}{c}\text { Camada } \\
(\mathrm{cm})\end{array}$ & $\begin{array}{c}\mathrm{Ks} \\
(\mathrm{cm} / \mathrm{h})\end{array}$ \\
\hline $0-10$ & 2,17 & $20-30$ & 1,32 \\
$10-20$ & 3,57 & $30-40$ & 3,21 \\
\hline
\end{tabular}

Clima: O clima da região foi denominado tipo CWA (subtropical úmido com estiagens no verão) segundo a classificação climática de Köppen, com a temperatura do mês mais frio inferior a $18^{\circ} \mathrm{C}$ e no mês mais quente superior a $22^{\circ} \mathrm{C}$. Este clima foi também classificado por CAMARGO et al. (1974) como Tropical de Altitude.

Os dados climáticos locais durante o período em que foi conduzido o ensaio foram obtidos junto ao Departamento de Física e Meteorologia da ESALQ/USP e são apresentados na TABELA 4. Estes dados referem-se à valores médios para períodos de dez dias (decêndios), sendo que o último período representa a média de onze dias.

TABELA 4 - Elementos do clima local. Valores médios por decêndio, para o período experimental.

\begin{tabular}{lccccccc}
\hline Decêndio & $\begin{array}{c}\text { Ins. } \\
\text { (h/dia) }\end{array}$ & $\begin{array}{c}\text { Prec. } \\
\text { (mm) }\end{array}$ & $\begin{array}{c}\text { Ur } \\
\%\end{array}$ & $\begin{array}{c}\text { Vento } \\
\text { (km/dia) }\end{array}$ & $\begin{array}{c}\text { Temp } \\
\left({ }^{\circ} \mathrm{C}\right)\end{array}$ & $\begin{array}{c}\text { ECA } \\
\text { (mm/dia) }\end{array}$ \\
\hline I * & 9,50 & 0,00 & 66,00 & 132,02 & 18,69 & 4,90 \\
II & 7,98 & 0,00 & 55,00 & 83,11 & 21,97 & 5,19 \\
III & 7,94 & 0,00 & 60,00 & 160,35 & 19,55 & 5,69 \\
IV & 7,65 & 15,10 & 59,00 & 109,65 & 21,41 & 5,69 \\
V & 5,86 & 53,80 & 74,00 & 117,43 & 21,37 & 4,69 \\
VI & 2,46 & 45,70 & 81,00 & 137,99 & 20,02 & 3,34 \\
VII & 9,52 & 13,60 & 67,00 & 113,37 & 23,55 & 6,98 \\
VIII & 8,99 & 0,00 & 60,00 & 85,04 & 24,75 & 7,27 \\
IX & 7,04 & 13,90 & 72,00 & 141,48 & 23,25 & 6,22 \\
\hline
\end{tabular}

* 10 dias após a semeadura, quando se deu a emergência

Planta: A cultura utilizada foi a cenoura (Daucus carota, L.), cultivar Nantes Superior, recomendada pelo Instituto Agronômico de Campinas - IAC (CAMARGO, 1984) para o período em que se realizou o experimento, tendo esta apresentado um ciclo fenológico de 101 dias. Dentro dos canteiros foi utilizada uma densidade de plantio na ordem de 20 plantas por metro linear, após os desbastes. No presente trabalho, foram realizados 2 desbastes.

Componentes da equação do Balanço Hídrico: Para efeito do balanço hídrico, há a necessidade de se considerar a profundidade para o volume de controle de solo $(\delta z)$, que neste caso corresponde à profundidade efetiva do sistema radicular (z). Nesta situação, $\mathrm{z}$ foi adotado como sendo igual à 0,25 metros.

O componente "variação do armazenamento de água" no solo foi estimado a partir de valores de umidade amostrados em perfis consecutivos de solo, obtidos indiretamente mediante a determinação do potencial mátrico, onde foi utilizada a curva de retenção da água no 
solo. $\mathrm{Na}$ área onde realizou-se o experimento foram instaladas três baterias de tensiômetros com manômetros de mercúrio, nas profundidades de 10 , 20, 30 e 40 centímetros.

Estas baterias localizavam-se inseridas nos segundo, terceiro e quarto canteiros, respectivamente.

Para estimativa do armazenamento de água no solo (A) foi utilizada a seguinte expressão:

$$
A=\int_{0}^{L} \theta \cdot d z=\bar{\theta} \cdot z
$$

sendo $\theta$, a umidade média do perfil do solo até a profundidade considerada (z). Neste estudo, a variação de armazenamento $(\Delta \mathrm{A})$ para um determinado intervalo de tempo (t1-to) correspondeu à diferença no teor de água do solo entre dias consecutivos.

O componente "precipitação pluviométrica" foi obtido a partir de dados coletados junto ao Departamento de Física e Meteorologia da ESALQ/ USP, cujo posto meteorológico situa-se à aproximadamente 870 metros do local do experimento.

Para o processo de irrigação da área experimental (estimativa do componente "irrigação" na equação de balanço hídrico) foi utilizado o método de aspersão convencional, com frequência diária de aplicação de água até o $40^{\circ}$ dia após o plantio. Esta metodologia foi utilizada seguindo-se recomendações técnicas adotadas por SILVA et al. (1982), onde é destacado que, para a ocorrência de boa germinação e emergência da cultura, as irrigações devem ser leves e frequentes, visando evitar principalmente a formação de crostas na superfície do terreno.

No caso, foram aplicados diariamente 4 $\mathrm{mm}$ de lâmina de água. Após este período, a irrigação tornou-se variável até o final do ciclo.

O controle das irrigações foi feito com base nas leituras médias dos tensiômetros localizados à 0,20 e 0,30 metros de profundidade, sempre que o potencial mátrico atingia um valor de 0,04 MPa.

A lâmina líquida de irrigação no período, já que o sistema de aspersão não apresentava eficiência de $100 \%$, foi representada pela média de 12 (doze) coletores uniformemente distribuídos na área. Estes coletores localizavam-se nas entrelinhas dos canteiros 2, 3, 4 e 5, espaçados de 3 metros, num total de 3 coletores por cada entrelinha.

O componente "drenagem profunda" e "ascenção capilar" foram estimados pelo fluxo de água no solo (qz), na profundidade e tempo considerado, utilizando-se a equação de Buckingham-Darcy:

$$
q_{z}=-K(\theta) \cdot \frac{\delta H}{\delta z}
$$

onde $\mathrm{K}(\theta)$ representa a condutividade hidráulica em relação a umidade do solo e $\delta \mathrm{H} / \delta \mathrm{z} \circ$ gradiente de potencial total da água na profundidade z. O sinal negativo indica que o sentido do fluxo de água é do maior para o menor potencial, estimados a partir das leituras dos tensiômetros.

O componente "deflúvio superficial" foi desconsiderado neste estudo devido à dificuldade da determinação do run-off que ocorreu na área, fato este presente somente nos períodos chuvosos.

Com relação às irrigações verificou-se não terem atingindo valores que viessem a favorecer o escoamento superficial, haja visto que os canteiros de plantio estavam nivelados. Este componente, como destaca REICHARDT (1985) é de difícil determinação ao nível de campo. Cita ainda REICHARDT (1986) que na equação do balanço hídrico, o "run-off" $\epsilon$ entendido como sendo uma incógnita, necessitando-se conhecer todos os outros componentes para sua estimativa.

O componente "evapotranspiração", cuja acumulação durante todo o ciclo corresponde à demanda de água da cultura, foi estimado por diferença dos outros componentes na equação 1 .

\section{Determinação da Evapotranspiração de}

Referência: Para a estimativa da evapotranspiração de referência, foi utilizado o método de Penman, onde são considerados os parâmetros de balanço de energia radiante e os princípios aerodinâmicos, propostos a partir do seguinte modelo (PENMAN, 1956):

$$
\text { Eto }=\frac{\Delta / \gamma \cdot H+E a}{\Delta / \gamma+1}
$$

sendo, $\Delta / \gamma$ uma constante determinada em função da temperatura média do ar, $\mathrm{H}$ o balanço de energia diário, determinado pela expressão: 


$$
\begin{aligned}
H= & \frac{Q 0}{59} \cdot\left(a+b \cdot \frac{n}{N}\right) \cdot(1-r)- \\
& -\frac{\sigma \cdot T a^{4}}{59} \cdot(0,56-0,09 . \\
& \cdot \sqrt{e a}) \cdot\left(0,1+0,9 \cdot \frac{n}{N}\right)
\end{aligned}
$$

sendo Q0/59 a radiação solar global na ausência da atmosfera, em função da latitude e época do ano, a e b parâmetros locais definidos para Piracicaba por OMETTO (1981) como sendo iguais a $0,26 \mathrm{e}$ 0,51 , respectivamente, $\mathrm{n}$ a insolação média real, $\mathrm{N}$ $a$ insolação máxima diária, $r$ o poder refletor da superfície (albedo), tomado como sendo 0,25 para a superfície gramada, $\sigma$ a constante de StefanBolztman, Ta a temperatura da superfície e ea o valor médio diário da pressão de vapor da água.

$O$ parâmetro $\mathrm{Ea}$ corresponde ao poder evaporante do ar, obtido pela equação:

$$
E a=0,35 \cdot\left(1+\frac{U}{160}\right) \cdot(e s-e a)
$$

sendo $U$ a velocidade média do vento $(\mathrm{Km} / \mathrm{dia})$, obtida à 2 metros de altura e es a tensão de saturação da água.

Determinação do Coeficiente de Cultura: A determinação da evapotranspiração potencial para uma dada cultura de referência simplifica a estimativa da evapotranspiração de outras culturas irrigadas nos diferentes estádios fenológicos, atraveś de relações empíricas denominadas coeficientes de cultura $(\mathrm{Kc})$ (SAAD \& SCALOPPI, 1988). Este é um coeficiente adimensional sendo dado pela razão entre a evapotranspiração máxima de uma cultura $(E t m)$, cultivada sem restrição de água e a evapotranspiração de referência (Eto).

No presente estudo, os valores de $\mathrm{Kc}$ foram calculados para períodos decendiais (10 dias).

Utilizando-se da metodologia descrita foi realizado o experimento ao nível de campo visando a obtenção dos dados de demanda hídrica da referida cultura, cujos valores encontrados são apresentados e discutidos a seguir.

\section{RESULTADOS E DISCUSSÕES}

Os valores de evapotranspiraçãomáxima (Etm) estimados através do método do balanço hídrico foram obtidos num periodo de 91 (noventa e um) dias', a partir do décimo dia após o plantio. Portanto, o ciclo fenológico da cultura foi de 101 (cento e um) dias.

Estes valores foram divididos em 09 (nove) subperíodos de 10 (dez) dias (decêndio), sendo o último subperíodo de 11 (onze) dias, cujos dados são apresentados na TABELA 5:

\begin{tabular}{|c|c|c|c|c|c|c|}
\hline $\begin{array}{l}\text { Decên- } \\
\text { dio }\end{array}$ & $\begin{array}{r}\Delta A \\
(\mathrm{~mm})\end{array}$ & $\underset{(\mathrm{mm})}{\mathbf{P}}$ & $\underset{(\mathrm{mm})}{\mathbf{I}}$ & $\underset{(\mathrm{mm})}{\mathbf{q z}} \quad \mathbf{E t m}$ & méd. & $\begin{array}{c}\text { Etm acum. } \\
(\mathrm{mm})\end{array}$ \\
\hline I & 0,993 & 0,00 & 15,84 & $4-2,097$ & 1,27 & 12,70 \\
\hline II & 0,996 & 0,00 & 25,98 & $8-0,422$ & 2,46 & 37,30 \\
\hline III & 2,415 & 0,00 & 29,28 & $8-0,706$ & 2,62 & 63,50 \\
\hline IV & $+2,035$ & 15,10 & 35,20 & $0-1,037$ & 5,13 & 114,80 \\
\hline $\mathbf{V}$ & - 6,248 & 53,80 & 16,55 & $5-1,906$ & 6,22 & 177,00 \\
\hline VI & $+4,318$ & 45,70 & 0,00 & $-3,568$ & 4,65 & 223,50 \\
\hline VII & $+10,84$ & 13,60 & 27,41 & $1-1,383$ & 5,05 & 274,00 \\
\hline VIII & $+4,373$ & 0,00 & 47,88 & $8-0,059$ & 5,22 & 326,20 \\
\hline DX & $-7,616$ & 13,90 & 33,74 & $4-1,215$ & 3,53 & 365,03 \\
\hline
\end{tabular}

TABELA 5 - Valores médios da Etm por decêndio, determinados pelo método do balanço hídrico.

TTL + 3,304 142,10 231,88-12,393 365,03

Durante o período em que foi conduzido o experimento, os parâmetros que representaram a contribuição de água foram a precipitação $(P)$ e irrigação (I).

Através da TABELA 5 observa-se que as precipitaçōes ocorridas contribuiram para um aumento no valor estimado de $\mathrm{Etm}$, durante o quarto e quinto decêndios. COSTA (1986) ressalta dificuldades na determinação da Etm para períodos com ocorrência de precipitações, onde é destacado que a limitação de estimativas de evapotranspiração sob tais condições atmosféricas está correlacionada com a dificuldade para medir mudanças na lâmina armazenada no solo com precisão maior que \pm 2 $\mathrm{mm}$ de água, impedindo assim 0 uso de determinações diárias de evapotranspiração.

E importante destacar que a não contabilização de componente "deflúvio superficial" que pode ter ocorrido nos períodos chuvosos, é um fator que provavelmente provocou a superestimativa dos valores de Etm média ocorrida nos períodos em questão.

Com relação aos parâmetros que representam a contribuição de água ao meio, destaca-se que o controle feito durante todas as irrigações através de coletores uniformemente 
distribuidos na área permitiu a confiabilidade deste componente na equação do balanço hídrico. Erros inerentes a estes componentes variam de 1 a $5 \%$ o que mostra a necessidade de uso mais criterioso da técnica empregada (DOWNEY, 1972).

Os valores de perda de água por drenagem profunda (qz), comparados com os valores das precipitações ocorridas, foram considerados pequenos. $O$ uso da equação de Buckingham-Darcy para a estimativa do fluxo de água em condições de campo, exige o conhecimento de parâmetros que sofrem variações espaciais no solo, podendo levar a erros na estimativa deste componente.

Foi observado ainda que durante alguns dias, o fluxo de água apresentou resultados positivos, indicando a ocorrência de ascenção capilar. No entanto, para efeito do balanço hídrico, foi considerado o somatório dos componentes ascenção capilar e drenagem profunda ocorridas no decêndio, onde o fluxo resultante foi sempre a drenagem profunda.

Quanto a estimativa do armazenamento de água no solo $(\Delta \mathrm{A})$, observa-se que este componente apresentou uma variação na ordem de $-10,846$ a $+7,616 \mathrm{~mm}$, o que mostra que em alguns decêndios ocorreu perda de água no solo, ou seja, o consumo de água foi superior a quantidade aplicada $(\mathrm{P}+\mathrm{I})$. Neste contexto, o valor do consumo total durante o ciclo foi de $365,03 \mathrm{~mm} \mathrm{e}$ o valor médio de Etm no período foi de 3,61 $\mathrm{mm} /$ dia,

Da mesma forma que a evapotranspiração máxima medida pelo método do balanço hídrico, a evapotranspiração de referência foi agrupada em subperíodos decendiais, cujos valores são apresentados na TABELA 6. Esta TABELA apresenta informações sobre os valores estimados dos parâmetros a serem utilizados na equação de Pennam para a época em que foi realizado o experimento.

A partir desse dados, foi possível estimar os coeficientes de cultura (Kc), correlacionando os valores de Etm com os valores de Eto, para cada decêndio estudado, cujos resultados são apresentados na TABELA 7.

Nestas condições, o coeficiente de cultura médio encontrado para todo o ciclo vegetativo foi de 1,1. Pela TABELA 7 observa-se o efeito do valor superestimado de Etm medido em períodos chuvosos sobre a provável superestimativa do coeficiente de cultura (quinto decêndio).
TABELA 6 - Evapotranspiração de referência segundo o método de Penman.

Decên- ea oTa'/59 Rs $\mathrm{H} \quad \Delta / \gamma \quad \mathrm{Ea} \quad$ Eto

dio (mmhg) (mmeq) (mmeq) (mmeq) (mmeq) (mm)

\begin{tabular}{lrrrrrrr}
\hline I & 10,62 & 14,60 & 7,97 & 2,60 & 2,00 & 3,54 & 2,92 \\
II & 10,97 & 15,27 & 7,17 & 2,40 & 2,60 & 4,71 & 3,05 \\
III & 10,20 & 14,77 & 8,13 & 3,30 & 2,00 & 4,84 & 3,81 \\
IV & 11,29 & 15,15 & 7,96 & 3,34 & 2,36 & 4,61 & 3,72 \\
V & 14,16 & 15,14 & 6,93 & 3,39 & 2,35 & 3,00 & 3,27 \\
VI & 14,21 & 14,87 & 5,63 & 3,32 & 2,00 & 2,17 & 2,94 \\
VII & 14,53 & 15,60 & 10,08 & 4,93 & 2,60 & 4,38 & 4,78 \\
VIII & 14,12 & 15,85 & 9,74 & 4,71 & 2,75 & 5,00 & 4,79 \\
IX & 15,42 & 15,54 & 9,05 & 4,91 & 2,60 & 3,97 & 4,65
\end{tabular}

TABELA 7 - Valores de coeficiente de cultura (Kc) estimados por decêndio.

\begin{tabular}{lccc}
\hline Decêndio & $\begin{array}{c}\text { Etm } \\
\text { (mm/dia) }\end{array}$ & $\begin{array}{c}\text { Eto } \\
\text { (mm/dia) }\end{array}$ & Kc \\
\hline I & 1,27 & 2,92 & 0,43 \\
II & 2,46 & 3,05 & 0,80 \\
III & 2,62 & 3,81 & 0,68 \\
IV & 5,13 & 3,72 & 1,37 \\
V & 6,22 & 3,27 & 1,90 \\
VI & 4,65 & 2,94 & 1,58 \\
VII & 5,05 & 4,78 & 1,05 \\
VIII & 5,22 & 4,79 & 1,09 \\
IX & 3,53 & 4,65 & 0,76 \\
\hline
\end{tabular}

Para uma maior adequação ao manejo da irrigação, estes coeficientes foram agrupados de acordo com a fase de desenvolvimento da cultura, sendo apresentados pela TABELA 8. DOOREMBOS \& PRUITT (1977) recomendam a adoção de valores de Kc para diferentes estádios de desenvolvimento da cenoura na seguinte forma: etapa inicial (20 a 30 dias), etapa de desenvolvimento (30 a 40 dias), média estação (30 a 80 dias) e etapa final (20 dias).

A TABELA 8 apresenta ainda os valores de Kc propostos por DOOREMBOS \& PRUITT (1977).

Nota-se que os coeficientes de cultura obtidos apresentaram certa variabilidade com relação aos propostos pela FAO. Observa-se uma tendência de crescimento para os estádios de médio e final, o que pode ser explicado pela limitação do método à períodos chuvosos, devido à dificuldade da contabilização do componente deflúvio 
superficial na equação do balanço hídrico, como já destacado por REICHARDT $(1985,1986)$.

TABELA 8 - Valores de coeficientes de cultura por fase de desenvolvimento vegetativo.

\begin{tabular}{lcc}
\hline Fases & Kc obtido & Kc FAO \\
\hline Inicial & 0,54 & $0,90-0,95$ \\
Desenvolvimento & 0,97 & $0,97-1,07$ \\
Médio & 1,52 & $1,00-1,15$ \\
Final & 0,93 & $0,70-0,85$
\end{tabular}

* Kc FAO corresponde aos coeficientes propostos por DOOREMBOS \& PRUITT (1977)

ARAGÃO JÚNIOR (1982) determinou os valores de Kc para a mesma cultura utilizando - método do balanço hídrico sob condições de campo, em Guaramiranga (CE), considerando três estádios de desenvolvimento. Os resultados obtidos mostraram-se coerentes com os apresentados por DOOREMBOS \& PRUITT (1977) para os dois últimos estádios: 0,99 e 0,86, respectivamente.

A variabilidade com relação aos resultados encontrados neste trabalho com o desenvolvido por ARAGÃO JÚNIOR (1982) pode ser explicada pelas diferenças climáticas encontradas nas duas regiões em estudo, fato este que salienta a importância das determinações de consumo hídrico e Kc para um lugar específico.

\section{CONCLUSÕES}

De posse dos resultados obtidos para as condições em que foi desenvolvido o experimento, pode-se concluir que 0 balanço hídrico, sob condições de campo, apresentou resultados que viabilizam sua utilização na determinação da demanda hídrica de uma cultura, comparando-se com valores estabelecidos na literatura. Para a cultura da cenoura, o consumo total de água, considerando-se um ciclo vegetativo de 101 dias, foi de $365,03 \mathrm{~mm}$, gerando um consumo médio de $3,61 \mathrm{~mm} /$ dia. Nesta situação o coeficiente de cultura encontrado foi de 1,1 .

Entretanto, é importante destacar a limitação dessa metodologia de estimativa quando da não contabilização do componente "deflúvio superficial" na equação do balanço hídrico, componente este de difícil determinação ao nível de campo.

\section{REFERÊNCIAS BIBLIOGRÁFICAS}

ARAGÃo JÚNIOR, T.C. Determinação do coeficiente de cultura $(\mathrm{Kc})$ para a cenoura (Daucus carota, $L$.) pelo método do balanço hídrico. Fortaleza, 1982. 45p. Dissertação (Mestrado) - Universidade Federal do Ceará.

CAMARGO, A.P. de; PINTO, H.S.; BRUNINI, O.; PEDRO JÚNIOR, M.J.; ORTOLANI, A.A.; ALFONSI, R.F. Clima do Estado de São Paulo. In SÃO PAULO. Secretaria da Agricultura. Zoneamento agrícola do Estado de São Paulo. São Paulo, 1974. v.1, p.51-87.

CAMARGO, L. S. As hortaliças e seu cultivo. 2.ed. Campinas, Fundação Cargill, 1984. 448p.

COSTA, A.C.S. da. Balanço hídrico em culturas de feijāo (Phaseolus vulgaris, L.) e do milho (Zea mays, L.) sob condições de campo. Piracicaba, 1986. 166p. Dissertação (Mestrado) - Escola Superior de Agricultura "Luiz de Queiroz", Universidade de São Paulo.

DOOREMBOS, J.; PRUITT, W.O. Guidelines for predicting crop water requirements. Rome, FAO, 1977. 179p. (FAO. Irrigation and Drainage Paper, 24).

DOWNEY, L.A. Water-yield relation for nonforage crops. Journal of the Irrigation and Drainage Division, Ney York, v.98, p.107-115, 1972.

FRIZZONE, J.A. Funções de resposta do feijoeiro (Phaseolus vulgaris, L.) ao uso de nitrogênio e lâmina de irrigação. Piracicaba, 1986. Tese (Doutorado) Escola Superior de Agricultura "Luiz de Queiroz", Universidade de São Paulo.

MAROUELLI, W.A.; VIEIRA, J.V. A irrigaçāo na produção de raizes e sementes de cenoura. ITEM, Brasília, v.42, p.29-31, 1990.

OMETTO, J.C. Bioclimatologia vegetal. São Paulo, Editora Agronômica Ceres, 1981. 440p.

PENNAM, H.L. Evaporation: an introducting survey. Netherlands. Journal of Agricultural Science, Cambridge, v.4, p.9-29, 1956.

RANZANI, G.O.; FREIRE, O.; KINJO, T. Carta de solos do município de Piracicaba. Piracicaba, ESALQ, 1966. 95p.

REICHARDT, $K$. Processos de transferência no sistema solo-planta-atmosfera. Campinas: Fundação Cargill, 1985. 486p. 
REICHARDT, K. Água em sistemas agrícolas. São Paulo: Manole, 1986. 188p.

REICHARDT, K.; LIBARDI, P.L.; SAUNDERS, L.C.U. Dinâmica da água $\mathrm{em}$ solos cultivados com milho. Revista Brasileira de Ciência do Solo, Campinas, v.3, p.1-5, 1989.

ROSE, C.W.; STERN, W.R. Determination of withdrawal of water from soil by crop roots as a function of depth and time. Australian Journal Soil Research, East Melbourne, v.5, p.11-19. 1969.

SAAD, A.M. Uso do tensiômetro no controle da irrigação por pivo central em cultura do feijoeiro (Phaseolus vulgaris, L.). Piracicaba, 1991. 144p. Dissertação (Mestrado) - Escola Superior de Agricultura "Luiz de Queiroz", Universidade de São Paulo.

SAAD, J.C.C.; SCALOPPI, E.J. Análise dos principais métodos climatológicos para estimativa da evapotranspiraçāo. In: CONGRESSO NACIONAL DE IRRIGAÇÃO E DRENAGEM, 8., 1988. Florianópolis. Anais. Florianópolis: ABID, 1988. v.2, p.999-1021.
SILVA, W.L.C.; VIEIRA, J.V.; CARRUO, D.A. Efeito de diferentes tensōes de umidade do solo sobre a cultura da cenoura em fase de desenvolvimento ativo do sistema radicular. Pesquisa Agropecuária Brasileira, Brasília, v.17, n.2, p.211-214, 1982.

VAN GENUCHTEN, M.Th. A closed-form equation for predicting the hidraulic conductivity of unsaturated soil. Soil Science Society America Journal, Madison, v.44, p.892-898, 1980.

Enviado para publicação em $\mathbf{1 3 . 1 2 . 9 3}$

Aceito para publicação em 05.07 .94 